\title{
Application of a Potentiostatic Coulometric Titration Technique for an Oxygen Analyzer
}

\author{
By Shinya Otsuka* and Zensaku Kozuka*
}

\begin{abstract}
A new type of oxygen analyzer based on strict theoretical considerations was constructed utilizing the electrochemical oxygen pump: $\mathrm{Pt}$, $\mathrm{Air} / \mathrm{ZrO}_{2}\left(+\mathrm{Y}_{2} \mathrm{O}_{3}\right) / \mathrm{Ar}+\mathrm{O}_{2}$ or $\mathrm{Ar}+\mathrm{H}_{2}+\mathrm{H}_{2} \mathrm{O}$ gas mixture, Pt. Various amounts of air were injected into a circulating $\mathrm{Ar}$ or $\mathrm{Ar}+\mathrm{H}_{2}$ gas mixture, while lead samples were melted in the gas mixture. The oxygen content was determined within $0.18 \mathrm{ks}$ from the change in the electrical current passing through the cell. This technique overcame errors due to the release of oxygen from the electrolyte and electronic conduction in the electrolyte. Neither standard samples nor the establishment of blank conditions was necessary. The analytical values for air were in excellent agreement with the theoretical ones. The analytical values for lead were also highly consistent and reliable despite the fairly low oxygen concentrations in the samples. This type of oxygen analyzer appears to be useful for determining oxygen concentrations less than 1 mass ppm.
\end{abstract}

(Received March 7, 1984)

Keyzords: oxygen analyzer, coulometric titration, potentiostatic experiment, solid electrolyte, stabilized zirconia

\section{Introduction}

In the last decade, the effects of nonmetallic elements, e.g., oxygen, sulfur, carbon, nitrogen and hydrogen, on the quality of ferrous and nonferrous materials are topics of growing technological interest ${ }^{(1)(2)}$. Advanced techniques to control and analyze the amounts of these impurities have became increasingly necessary. In previous papers ${ }^{(3)(4)}$, the present authors proposed a modified coulometric titration method employing a solid electrolyte to determine the activity coefficient of oxygen, $\gamma_{0}$, in melts. Errors due to the electronic conduction in the solid electrolyte and the release of oxygen from the electrolyte were evaluated and subtracted. As a result, fairly low oxygen concentrations in various liquid metals ${ }^{(5)(6)}$, alloys ${ }^{(5)(7)}$ and metal-nonmetal solutions ${ }^{(5)(8)}$ could be determined with a high accuracy as a function of the partial pressure of oxygen exerted by the dissolved oxygen in the melts. This type of potentiostatic coulometric titration techniques appears to be worthy of receiving an application as an oxygen analyzer. The objective of the present investigation is to develope an oxy-

* Department of Metallurgical Engineering, Faculty of Engineering, Osaka University, Suita, Osaka 565, Japan. gen analyzer with a high precision even when the oxygen is present in trace amounts. An oxygen analyzer constructed in this study requires neither standard samples nor the establishment of blank conditions. The performance of the apparatus was tested using various amounts of air and two types of lead. When the oxygen content is very low, the standard samples are not available, and therefore the oxygen analyzer used in this study may become more useful. In view of the fact that the performance of the apparatus cannot be tested for the very low oxygen content, the theoretical basis of the analyzer may be more important. With this in mind, in the present investigation, the principle of the modified coulometric titration which was a special case of the potentiostatic coulometric titrations was first newly established.

\section{A Modified Coulometric Titration Method}

\section{Ideal solid electrolyte ${ }^{\dagger}$}

In a series of the previous experiments for the determination of $\gamma_{0}$ in melts ${ }^{(4)-(8)}$, a cylindrical electrochemical cell with an air $\cdot \mathrm{Pt}$

$\dagger$ In the present paper, an ideal solid electrolyte has been defined as a solid electrolyte having an invariable composition during the experiments. 
reference electrode was subjected to potentiostatic pump-out operations. In order to simplify the following calculations, the one-dimensional cell (I) may be considered here:

$\mathrm{Pt}$, Air/Oxide Electrolyte/O in liquid metal, Cell (I)

$$
x=0 \quad x=L
$$

where the oxide electrolyte stands for a solid electrolyte, e.g., $\mathrm{ZrO}_{2}(+\mathrm{CaO}), \mathrm{ZrO}_{2}\left(+\mathrm{Y}_{2} \mathrm{O}_{3}\right)$, $\mathrm{ZrO}_{2}(+\mathrm{MgO})$ or $\mathrm{ThO}_{2}\left(+\mathrm{Y}_{2} \mathrm{O}_{3}\right)$. The initial open-circuit emf, $E_{1}(r)$, of the cell (I) is given by

$$
E_{1}(r)=-(F / 4)\left(\mu_{\mathrm{O}_{2}}^{1}-\mu_{\mathrm{O}_{2}}^{\circ}\right) \text {, }
$$

under the condition of $\bar{t}_{\text {ion }} \approx 1$, where $\mu_{\mathrm{O}_{2}}^{\circ}$ and $\mu_{\mathrm{O}_{2}}^{1}$ are the oxygen chemical potential in air and the initial oxygen chemical potential at the electrolyte-melt interface $x=L$, respectively, $F$ is the Faraday constant, and $\bar{t}_{\text {ion }}$ is the average ionic transference number of the electrolyte. A potentiostatic pump-out experiment was initiated by applying a voltage so that the oxygen chemical potential at $x=L$ was set to a constant value, $\mu_{\mathbf{O}_{2}}^{L}$. Under the condition of $\mu_{\mathbf{O}_{2}}^{L} \ll$ $\mu_{\mathrm{O}_{2}}^{1}$, the oxygen dissolved in the melt was transported electrochemically to the air $\cdot \mathrm{Pt}$ reference electrode.

The electrical current at a given time may be analyzed in terms of a mobil oxygen ion vacant site, $V_{\ddot{o}}$, in the solid electrolyte, following Wagner's theoretical treatment ${ }^{(9)-(13)}$. In the presence of an electrostatic field, the local equilibria at any location within the solid electrolyte are described by

and

$$
1 / 2 \mu_{\mathrm{O}_{2}}+\eta_{V \ddot{\mathrm{o}}}+2 \eta_{\ominus}=\mu_{\mathrm{O}_{\mathrm{o}} \mathrm{x}}
$$

$$
\alpha(t)=\frac{1}{L} \int_{0}^{L}\left\{\sigma_{\ominus}^{\circ} \exp \left[\left(\mu_{\mathrm{O}_{2}}^{\circ}-\mu_{\mathrm{O}_{2}}\right) / 4 R T\right]+\sigma_{\oplus}^{\circ} \exp \left[\left(\mu_{\mathrm{O}_{2}}-\mu_{\mathrm{O}_{2}}^{\circ}\right) / 4 R T\right]\right\} \partial x
$$

and

$$
\beta=\sigma_{\ominus}^{\circ}\left\{1-\exp \left[\left(\mu_{\mathrm{O}_{2}}^{\circ}-\mu_{\mathrm{O}_{2}}^{L}\right) / 4 R T\right]\right\}+\sigma_{\oplus}^{\circ}\left\{\exp \left[\left(\mu_{\mathrm{O}_{2}}^{L}-\mu_{\mathrm{O}_{2}}^{\circ}\right) / 4 R T\right]-1\right\},
$$

where $\sigma_{\ominus}^{\circ}$ and $\sigma_{\oplus}^{\circ}$ are the partial conductivities of excess electrons and positive holes for $\mu_{\mathrm{O}_{2}}^{\circ}$. The second term in eq. (6), i.e., $I_{\mathrm{e}}$, is the electronic current which depends on $f(t)$ and time, $t$. The third term in eq. (6), i.e., $I_{\mathrm{e}}^{*}$, is the electronic current which is independent of $f(t)$ and $t$. When $I_{\text {ion }}=0$, i.e., $f(t)=0$, the total current $I$ reduces to $I_{\mathrm{e}}^{*} \cdot \alpha(t)$ corresponds to the where $\mu_{\mathrm{O}_{2}}$ is an oxygen chemical potential, $\eta_{V: \%}, \eta_{\ominus}$ and $\eta_{\oplus}$ are the electrochemical potentials of a vacant oxygen ion site, excess electrons and positive holes, respectively, and $\mu_{\mathrm{O}_{\mathrm{O}} \mathrm{X}}$ is the chemical potential of an oxygen ion on its site.

The partial current $I_{j}$ for species $j$ within the electrolyte is

$$
I_{j}=-\frac{\sigma_{j} A}{Z_{j} F} \frac{\partial \eta_{j}}{\partial x}
$$

where $A$ is the interfacial area between the electrolyte and melt, and $\sigma_{j}, \eta_{j}$ and $Z_{j}$ are the partial conductivity, electrochemical potential and valence, respectively, of the species $j$.

Because $\mu_{\mathrm{O}_{\mathrm{O}} \mathrm{X}}$ and $\mu_{V_{\ddot{o}}}$ are constant in the oxide electrolyte homogeneously doped with immobile cations ${ }^{(9)(10)}$, the value of $\partial \eta_{V: 0} / \partial x=$ $f(t)$ is independent of $x$. Differentiating eqs. (2) and (3) and substituting for eq. (4), one obtains

$$
\begin{aligned}
I & =I_{\text {ion }}+I_{\ominus}+I_{\oplus} \\
& =-\frac{\sigma_{\text {ion }} A}{2 F} f(t)-\left\{\frac{\sigma_{\ominus} A}{4 F}+\frac{\sigma_{\oplus} A}{4 F}\right\}\left\{\frac{\partial \mu_{\mathrm{O}_{2}}}{\partial x}+2 f(t)\right\},
\end{aligned}
$$

where $I$ is the total electrical current.

$\sigma_{\ominus}$ and $\sigma_{\oplus}$ are proportional to $\exp \left(-\mu_{\mathrm{O}_{2}} l\right.$ $4 R T)$ and $\exp \left(\mu_{\mathrm{O}_{2}} / 4 R T\right)$, respectively ${ }^{(9)}$, where $R$ is the gas constant and $T$ is temperature. In view of the fact that $I$ is independent of $x$, integrating $x=0$ to $L$, one obtains

$$
\begin{aligned}
I & =I_{\text {ion }}+I_{\mathrm{e}}+I_{\mathrm{e}}^{*} \\
& =-\frac{\sigma_{\text {ion }} A}{2 F} f(t)-\frac{A \alpha(t)}{2 F} f(t)-\frac{R T A \beta}{F L}
\end{aligned}
$$

with

average electronic conductivity of the electrolyte. Accordingly, the average ionic transference number of the electrolyte, $\bar{t}_{\text {ion }}$, at time $t$ is related to the $I_{\text {ion }}$ and $I_{\mathrm{e}}$ by the equation:

$$
\bar{t}_{\text {ion }}=\frac{I_{\text {ion }}}{I_{\text {ion }}+I_{\text {e }}}
$$




\section{Actual solid electrolyte}

When the oxygen chemical potential at the interface $x=L$ is changed from $\mu_{\mathrm{O}_{2}}^{1}$ to $\mu_{\mathrm{O}_{2}}^{L}$ by applying a voltage to the cell, a redistribution of oxygen chemical potential within the solid electrolyte takes place. This redistribution must be accompanied with the slight change in the concentration of a vacant oxygen ion site, $V_{\ddot{0}}$. This change may be virtually very small. However, the density of $V_{\ddot{o}}$ is extremely large. Additionally, any conventional solid electrolyte may include slight impurity oxides of $\mathrm{Si}, \mathrm{Fe}$ and so forth which would be deoxidized at a lower $\mu_{\mathrm{O}_{2}}$. Thus, as is observed in the previous experiments $^{(3)-(8)(14)(15)}$, the ionic current due to the oxygen released from the electrolyte cannot be neglected, provided the oxygen content in the melt is very low. On the basis of these considerations, the total current $I$ is expressed by the generalized equation,

$$
I=I_{\text {ion }}+I_{\text {ion }}^{\prime}+I_{\mathrm{e}}+I_{\mathrm{e}}^{\prime}+I_{\mathrm{e}}^{*},
$$

instead of eq. (6), where $I_{\text {ion }}$ and $I_{\text {ion }}^{\prime}$ are newly defined as the ionic currents due to the oxygen transferred from the melt and the oxygen released from the electrolyte, $I_{\mathrm{e}}$ and $I_{\mathrm{e}}^{\prime}$ are the electronic currents related to $I_{\text {ion }}$ and $I_{\text {ion }}^{\prime}$, respectively, and $I_{\mathrm{e}}^{*}$ has the same physical meaning as the one introduced in eq. (6). In view of eqs. (6) and (7), one obtains

$$
\bar{t}_{\text {ion }}=\frac{I_{\text {ion }}}{I_{\text {ion }}+I_{\mathrm{e}}}=\frac{I_{\text {ion }}^{\prime}}{I_{\text {ion }}^{\prime}+I_{\mathrm{e}}^{\prime}} .
$$

The electrical components passing through the cell (I) during the potentiostatic operation from $\mu_{\mathrm{O}_{2}}^{1}$ to $\mu_{\mathrm{O}_{2}}^{L}$ at $x=L$ are illustrated in Fig. 1 . Upon applying a voltage to the cell, the oxygen dissolved in the melt begins to be transferred to the air $\cdot \mathrm{Pt}$ reference electrode. With a decrease in the oxygen content in the melt, the total current decreases rapidly at first and then slowly with time. This situation is indicated in Fig. 1 as a state $A$. Then, in a state $B$, the current reaches a nearly constant value, $I_{\infty}$, which can be attributed mainly to $I_{\mathrm{e}}^{*}$ but still involves $I_{\mathrm{ion}}^{\prime}$ and $I_{\mathrm{e}}^{\prime}$. Later, the current $I_{\infty}$ finally decreases to the electronic component, $I_{\mathrm{e}}^{*}$, (state C). The total quantity of electrical charge due to $I_{\text {ion }}$, denoted by $Q_{\text {ion }}$, corresponds to the amount of

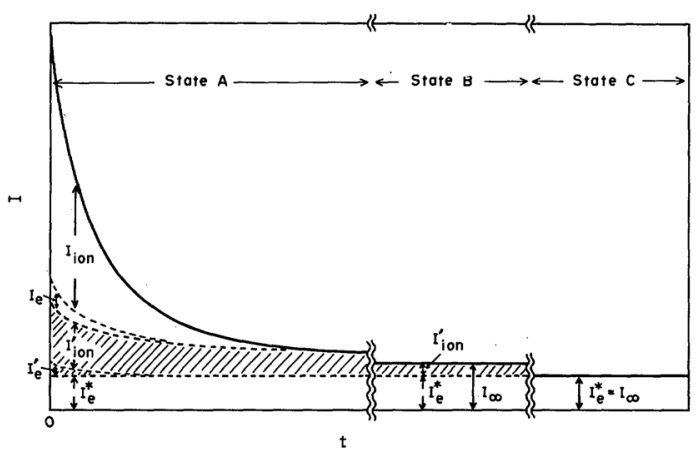

Fig. 1 A schematic representation of the decay curve of the electrical current with time during a potentiostatic coulometric titration from $\mu_{\mathbf{O}_{2}}^{1}$ to $\mu_{\mathbf{O}_{2}}^{L}$. $I_{\text {ion }}$ and $I_{e}$; the ionic and electronic currents due to oxygen transfer from the melt, $I_{\mathrm{ion}}^{\prime}$ and $I_{\mathrm{e}}^{\prime}$; the ionic and electronic currents due to the release of oxygen from the electrolyte, $I_{\mathrm{e}}^{*}$; the electronic current which is independent of time. $I_{\infty}$ corresponds to $\left(I_{\text {ion }}^{\prime}+\right.$ $\left.I_{\mathrm{e}}^{\prime}+I_{\mathrm{e}}^{*}\right)\left\{\approx\left(I_{\mathrm{ion}}^{\prime}+I_{\mathrm{e}}^{*}\right)\right\}$ in the state $\mathrm{B}$, and $I_{\mathrm{e}}^{*}$ in the state C. The shaded area indicates the electrical charge due to $\left(I_{\text {ion }}^{\prime}+I_{\mathrm{e}}^{\prime}\right)$.

oxygen which has been contained in the melt. As far as the potentiostatic pump-out operation is performed under the condition of $\bar{t}_{\text {ion }} \approx 1$, $I_{\mathrm{e}}$ and $I_{\mathrm{e}}^{\prime}$ can be neglected in comparison with $I_{\text {ion }}$ and $I_{\text {ion }}^{\prime}$.

Previously, Otsuka and co-workers ${ }^{(4)-(8)}$ carried out the modified coulometric titration experiments for the determination of $\gamma_{0}$ in various melts, in a manner described below. The nearly constant current, $I_{\infty}$, observed in the state B, was first subtracted from the total current $I$. The quantity of electrical charge due to the remaining current includes an error due to a part of $I_{\text {ion }}^{\prime}$. This error was evaluated by performing similar additional pump-out experiments in the range of negligibly low oxygen concentrations in the melts. Finally, the total quantity of electrical charge due to $I_{\text {ion }}$, denoted by $Q_{\text {ion }}$, could be determined as a function of the initial emf, $E_{1}(r)$. The details of the experimental procedures have been given in the previous papers $^{(4)(14)}$.

One may try to use this experimental technique as an oxygen analyzer. However, a major problem may come from the fact that the additional experiments to evaluate the error due to $I_{\text {ion }}^{\prime}$ take 4 or more ks. Furthermore, as the oxygen content in the melts becomes much lower, 
the procedure of subtracting this error appears to yield a less precise value of $Q_{\text {ion }}$. Thus, we should devise an alternative method to use as an oxygen analyzer with a high precision.

\section{An Oxygen Analyzer Utilizing a Potentiostatic Coulometric Titration Technique}

\section{Principle of the oxygen analyzer}

It is important to note that the complexity of the analytical procedure adopted in the modified coulometric titration experiments essentially comes from a change in $\mu_{\mathrm{O}_{2}}$ at $x=L$,

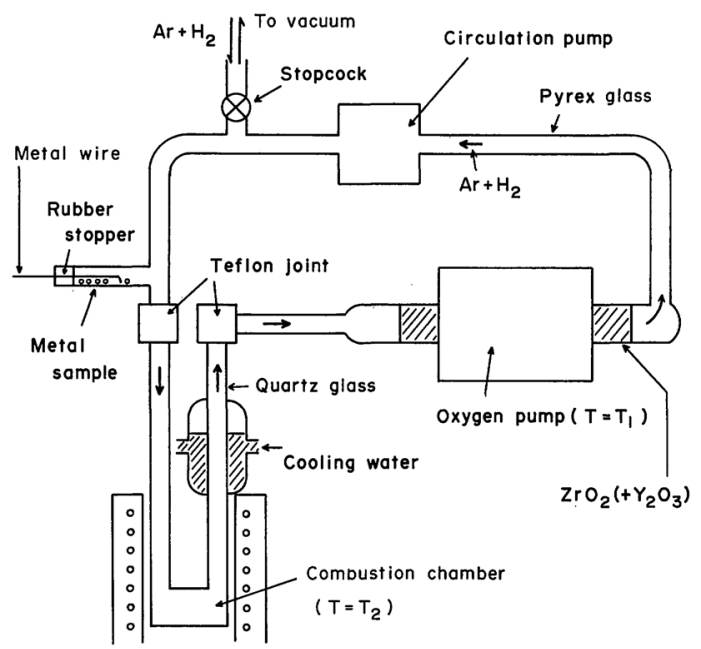

(a)

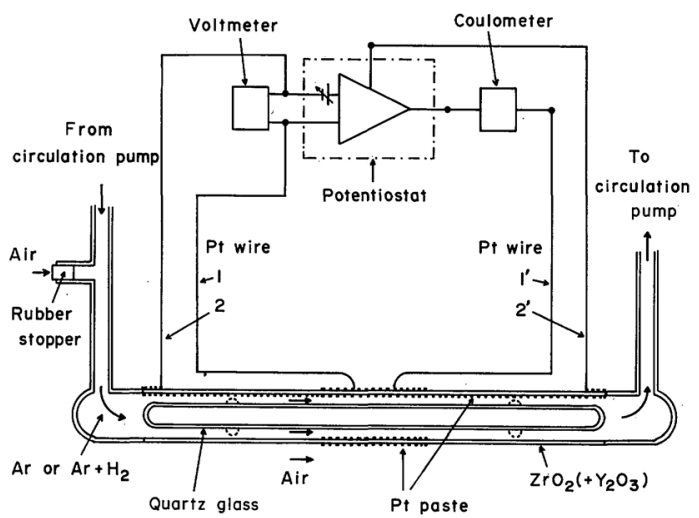

(b)

Fig. 2 Schematic drawings of the oxygen analyzer used in the present study, (a) for the analysis of lead, (b) for the analysis of air. i.e., from $\mu_{\mathrm{O}_{2}}^{1}$ to $\mu_{\mathrm{O}_{2}}^{L}$. Therefore, an attention is paid here to the state $\mathrm{B}$ or $\mathrm{C}$ as shown in Fig. 1, in which $\mu_{\mathrm{O}_{2}}$ at $x=L$ is always constant.

The oxygen analyzer of the present investigation, which was used for the analysis of lead, is schematically shown in Fig. 2(a). The analyzer contains an electrochemical oxygen pump:

$\mathrm{Pt}$, Air/Oxide Electrolyte/ $\mathrm{Ar}+\mathrm{H}_{2}+\mathrm{H}_{2} \mathrm{O}$ gas mixture, Pt.

Cell (II)

$$
x=0 \quad x=L,
$$

which is the same as that shown in Fig. 1(b). As indicated by an arrow in Fig. 1(a), this gas mixture is circulated throughout the closed system using a gas pump. A potentiostatic pump-out operation is performed by applying a voltage across wires $1^{\prime}$ and $2^{\prime}$ so that the emf across wires 1 and 2 has a preselected value, $E_{2}(m)$. The oxygen chemical potential at $x=L$ is set to a very low constant value, $\mu_{\mathbf{O}_{2}}^{L}$. After the state $\mathrm{B}$ is attained, i.e., $I=I_{\infty}$, a lead sample is dropped into the combustion chamber. As soon as the sample melts, the oxygen in it reacts with the circulating gas to form mainly $\mathrm{H}_{2} \mathrm{O}$ with some $\mathrm{O}_{2}$. When introduced into the cell (II), these gases lead to an increase in the electrical current, $I$. Because the gas mixture is

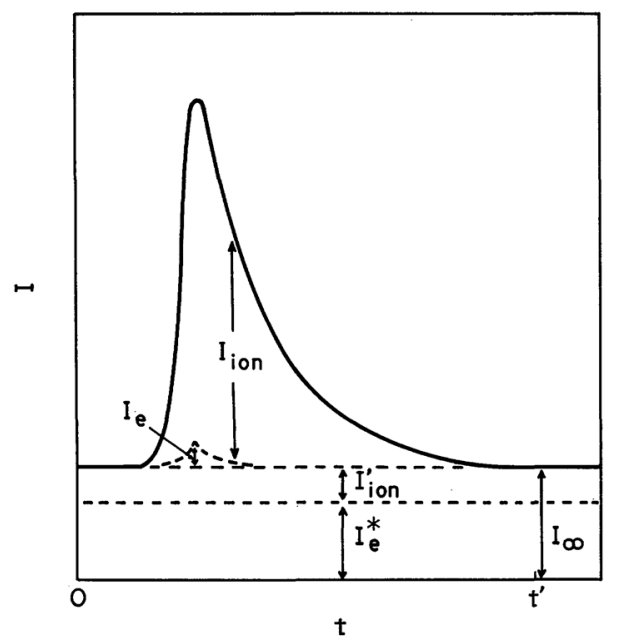

Fig. 3 A schematic representation of the electrical current versus time relationship observed when a sample is dropped into the combustion chamber. $I_{\text {ion }}$; the ionic current due to the oxygen evolved from the sample, $I_{\mathrm{e}}$; the electronic current which depends on $\boldsymbol{I}_{\text {ion }}, \boldsymbol{I}_{\infty}$; the background current, $\left(\boldsymbol{I}_{\text {ion }}^{\prime}+\right.$ $\left.I_{\mathrm{e}}^{\prime}+I_{\mathrm{e}}^{*}\right) \approx\left(I_{\mathrm{ion}}^{\prime}+I_{\mathrm{e}}^{*}\right)$. 
circulated repeatedly, the oxygen content of the sample becomes negligibly small. The oxygen chemical potential in the gas decreases to its starting value, $\mu_{\mathrm{O}_{2}}^{L}$. The electrical current $I$ also diminishes, until it reaches at $t=t^{\prime}$ the same value as the original background current $I_{\infty}$. The variation of the electrical current with time is schematically shown in Fig. 3. In the state B, the profile of the oxygen chemical potential near the grain-boundary in the electrolyte is independent of time. The oxygen transfer from the bulk to the grain-boundary is the rate-determining stage of $I_{\text {ion }}^{\prime}$. The passage of $I_{\text {ion }}$ has no influence on the $I_{\text {i on }}^{\prime}$. In view of eq. (9), when $I_{\mathrm{i} \text { on }}^{\prime}=$ constant, $I_{\mathrm{e}}^{\prime}$ is also virtually independent of time. Additionally, $I_{\mathrm{e}}^{\prime}$ may be negligibly small under the condition of $\bar{t}_{\text {ion }} \approx 1$. Because the background current, $I_{\infty}\left\{=I_{\text {ion }}^{\prime}+I_{\mathrm{e}}^{\prime}+I_{\mathrm{e}}^{*} \approx\right.$ $\left.I_{\mathrm{ion}}^{\prime}+I_{\mathrm{e}}^{*}\right\}$, is essentially constant, the increase in the current, as shown in Fig. 3, can be attributed to the electrical components $I_{\mathrm{ion}}$ and $I_{\mathrm{e}}$. The total quantity of electrical charge due to $I_{\text {ion }}$ corresponds to the amount of oxygen which the sample had contained.

Under the condition of $\bar{t}_{\text {ion }} \approx 1$, in view of eq. (9), $I_{\mathrm{e}}$ can be neglected in comparison with $I_{\text {ion }}$. Thus, one obtains

$$
I-I_{\infty}=I_{\text {ion }} .
$$

The total quantity of electrical charge due to $I_{\text {ion }}$, denoted by $Q_{\text {ion }}$, is given by

$$
\begin{aligned}
Q_{\text {ion }} & =\int_{0}^{t^{\prime}} I_{\text {ion }} \mathrm{d} t \\
& =\int_{0}^{t^{\prime}}\left(I-I_{\infty}\right) \mathrm{d} t .
\end{aligned}
$$

Finally, the mass of oxygen, $W_{\mathrm{O}}$, which the sample had contained, can be calculated using the equation:

$$
W_{\mathrm{O}}=\frac{M_{\mathrm{o}}}{2 F} Q_{\text {ion }},
$$

where $M_{\mathrm{O}}$ is the molar mass of oxygen.

The oxygen analysis may be performed, after the state $\mathrm{C}$ is attained. In this case, the background current $I_{\infty}$ is equivalent with $I_{\mathrm{e}}^{*}$. Accordingly, the $Q_{\text {ion }}$ value can be also calculated using eq. (11).

It should be emphasized that the simple eq. (11) was derived on a strict theoretical basis.
Any errors due to the electronic conduction in the solid electrolyte and the release of oxygen from the electrolyte can be overcome without any additional experiments. Thus, this technique should remarkably shorten the time spent for the oxygen analysis.

\section{Materials and equipment}

A $\mathrm{ZrO}_{2}\left(+8 \mathrm{~mol} \% \mathrm{Y}_{2} \mathrm{O}_{3}\right)$ solid electrolyte tube $(21 \mathrm{~mm}$ in o.d., $17 \mathrm{~mm}$ in i.d., and $500 \mathrm{~mm}$ long) was used as the electrolyte for the cell (II). This tube was supplied by Nippon Kagaku Togyo Co. Ltd. Platinum paste was applied to the inside and the outside surface of the tube over a $70 \mathrm{~mm}$ length at the midsection, for use as electrodes. The inside of the tube $(10 \mathrm{~mm}$ wide) as well as the outside ends of the tube was painted with platinum paste, as shown in Fig. 2(b), for use as leads. Four platinum lead wires were wounded around the outside painted surface. A quartz glass tube $(14 \mathrm{~mm}$ in o.d. and $500 \mathrm{~mm}$ long) was evacuated and sealed at both ends. This tube was placed inside the electrolyte to reduce the volume. The electrolyte tube was connected to a circulation pump (Misuzu model M-1500) using a Pyrex glass tube (4 $\mathrm{mm}$ in i.d.). A potentiostatic pump-out operation was performed using a potentiostat (Hokuto-Denko model PS-500B). The electrical current, $I$, and the electrical charge due to $I$, denoted by $Q_{T}$, were measured using a digital voltmeter (Yokogawa model 2502) and a coulometer (Hokuto-Denko model HF-201), respectively. $\mathrm{Ar}+\mathrm{H}_{2}$ gas mixtures were supplied by Seitetsu-Kagaku Co. Ltd.

Two types of lead rod (99.999 mass \% purity), designated $\mathrm{Pb}$ (I) and $\mathrm{Pb}$ (II), were obtained from different sources. As shown in Fig. 2(b), the combustion chamber constructed from a quartz glass tube $(18 \mathrm{~mm}$ in i.d. and $33 \mathrm{~mm}$ long) was installed between the electrolyte tube and the circulation pump. In order to protect the cell (II) from the very small lead vapors of the samples, a cooling water system was used between the cell (II) and the combustion chamber. When air was analyzed for oxygen, the combustion chamber and the cooling system were removed, as shown in Fig. 2(b). 


\section{Experimental procedure}

(1) Oxygen analysis of air

The electrochemical cell (II) was heated to $T_{1}=1073 \mathrm{~K}$. The closed system was first evacuated and then filled with an $\mathrm{Ar}, \mathrm{Ar}+0.0942 \%$ $\mathrm{H}_{2}$, or $\mathrm{Ar}+0.000702 \% \mathrm{H}_{2}$ gas mixture. The gas was circulated throughout the closed system at a flow rate of approximately $16 \mathrm{~cm}^{3} \cdot \mathrm{s}^{-1}$ using the circulation pump. When the potentiostatic pump-out operation was started, the electrical current first decreased rapidly and then slowly with time, until it finally reached a virtually constant value, $I_{\infty}$, in 12 or more ks. After that, a preselected amount of air was introduced into the closed system through a rubber stopper using an injector. By measuring the room temperature and atmospheric pressure, the amount of air injected was normalized to the volume, $V_{\text {Air }}$, at $273 \mathrm{~K}$ under $101325 \mathrm{~Pa}$. All $V_{\text {Air }}$ values reported in the present paper are normalized values. The electrical current $I$ first increased and then returned to the original value, $I_{\infty}$. The $Q_{\text {ion }}$ values were calculated from the measured values of $Q_{T}$ and $I_{\infty}$.

\section{(2) Oxygen analysis of lead}

The surface oxide of lead rod was first removed. Then, several pieces of these samples were weighed and placed inside the closed system at the position shown in Fig. 2(a). The temperature of the cell (II), $T_{1}$, was maintained at $1073 \mathrm{~K}$. The closed system was evacuated and filled with an $\mathrm{Ar}+0.0942 \% \mathrm{H}_{2}$ gas mixture. The combustion chamber was heated to $T_{2}=$ $1073 \mathrm{~K}$. After a virtually constant current, $I_{\infty}$, was attained under the condition of $E_{2}(m)=$ $1.3 \mathrm{~V}$, one of the lead samples was pushed forward using a metal wire, until the sample dropped into the combustion chamber. The electrical current first increased and then returned to its starting value, $I_{\infty}$, within $0.18 \mathrm{ks}$. Knowing the values of $Q_{T}$ and $I_{\infty}$, the $Q_{\text {ion }}$ value corresponding to the mass of oxygen which the sample had contained could be calculated according to eq. (11). After the oxygen analysis of the sample was completed, a subsequent analysis was performed by dropping another sample into the combustion chamber. The oxygen concentration in the lead rods used in the present investigation has not been determined by means of other oxygen analyzers. In order to check the reliability of the data obtained, the measured $Q_{\text {ion }}$ values were plotted as a function of the mass of the lead sample, $W_{\mathbf{P b}}$.

\section{Experimental results}

\section{(1) Oxygen analysis of air}

In the case of circulating Ar gas, the typical variation of the electrical current with time is presented in Fig. 4 for various amounts of air. At the time indicated by $\uparrow$, a preselected amount of air was injected into the closed system. As soon as a relatively small amount of air was injected, the electrical current increased rapidly and then smoothly decreased to the original value, $I_{\infty}$, within $0.18 \mathrm{ks}$. As a result, a peak on the current versus time curve, as indicated by A in Fig. 4, was observed. When the amount of air injected was relatively large, the second peak, as indicated by $\mathbf{B}$, followed the first peak. The appearance of the second peak reveals the fact that some amount of the air was not pumpedout electrochemically during the first passage through the electrolyte tube, and circulated repeatedly throughout the closed system. Even in this case, however, the electrical current decreased fairly smoothly to the original background current, $I_{\infty}$. If a larger amount of air was injected, the third peak on the curve might appear. Electrical current versus time curves for the circulating $\mathrm{Ar}+0.0942 \% \mathrm{H}_{2}$ and $\mathrm{Ar}+$ $0.000702 \% \mathrm{H}_{2}$ gas mixtures were also similar to those in Fig. 4.

The $Q_{\text {ion }}$ values calculated from the measured $Q_{T}$ and $I_{\infty}$ values are shown in Figs. 5 and 6 as a function of $V_{\text {Air }}$. The dotted line indicates the theoretical $Q_{\text {ion }}$ values. As seen from these figures, when the amount of air was relatively small, the measured $Q_{\text {ion }}$ values lay on the theoretical line. However, with the increase in $V_{\text {Air }}$, a slight deviation was observed. The injection of air leads to an increase in the total pressure of the circulating gas. As the amount of air was increased, a slight leakage of oxygen might occur through a pin hole of the rubber stopper. Accordingly, this deviation of the measured $Q_{\text {ion }}$ values from the theoretical line does not mean an essential problem of the oxygen analyzer used in this study. A trend 


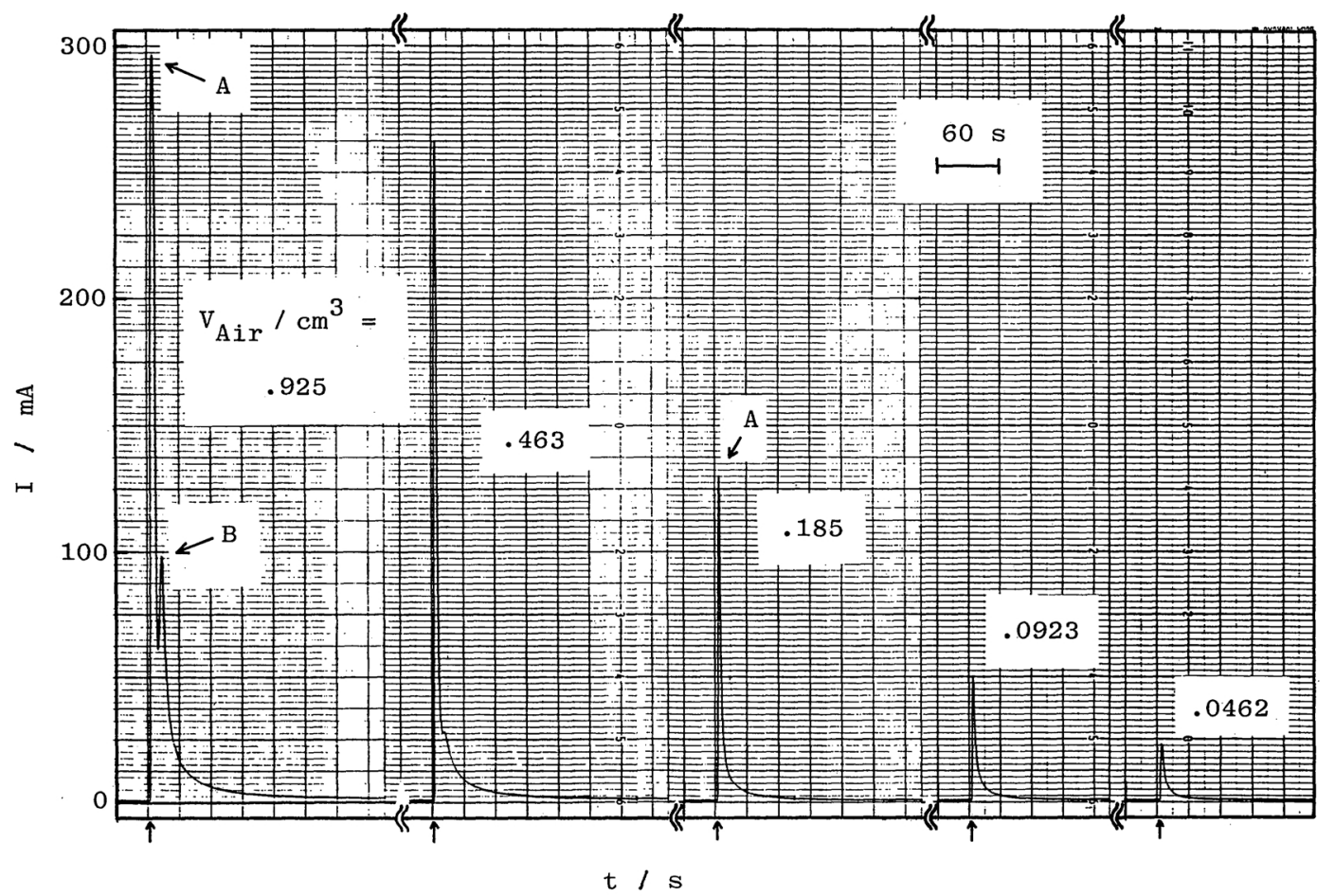

Fig. 4 Variation of the electrical current with time, for the air injected into a circulating Ar gas. The time at which the air was injected is indicated by $\uparrow$.

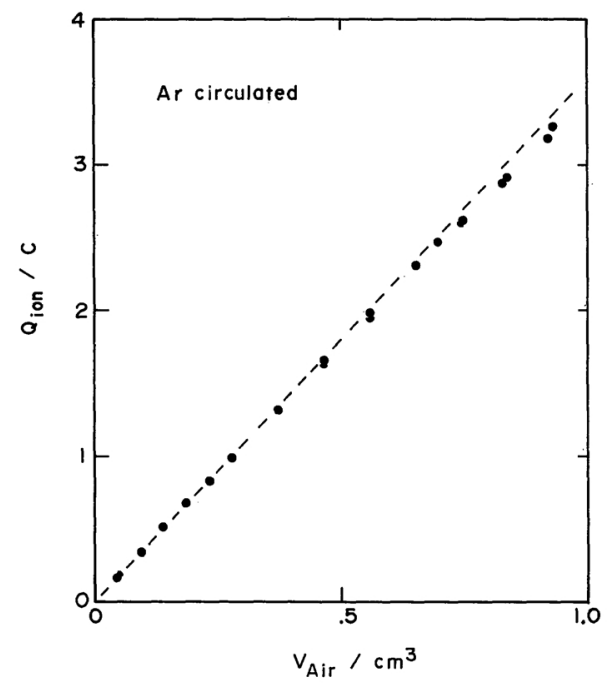

Fig. 5 Comparison of the measured $Q_{\text {ion }}$ values with the theoretical values as a function of $V_{\text {Air }}$, for a circulating Ar gas. 0 : measured values, -.-theoretical line.

similar to Figs. 5 and 6 was observed for the circulating $\mathrm{Ar}+0.000702 \% \mathrm{H}_{2}$ gas mixture.

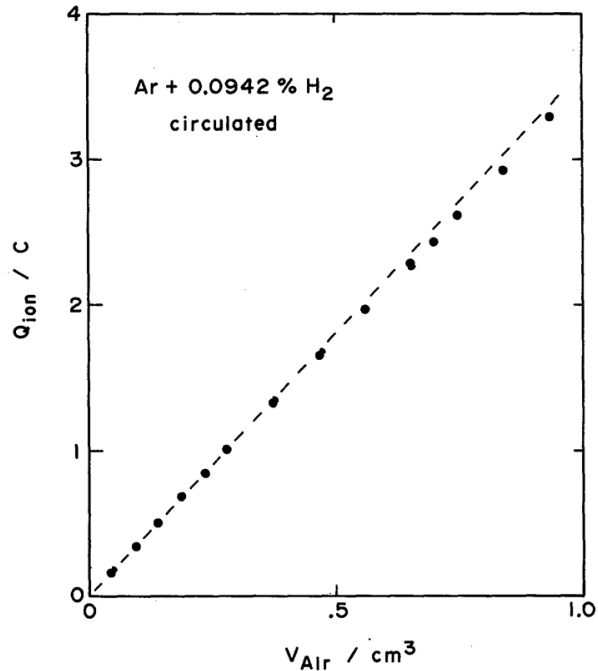

Fig. 6 Comparison of the measured $Q_{\text {ion }}$ values with the theoretical values as a function of $V_{\text {Air }}$, for a circulating $\mathrm{Ar}+0.0942 \% \mathrm{H}_{2}$ gas mixture. 0 : measured values, ----: theoretical line.

In Fig. 7, the measured $Q_{\text {ion }}$ values are compared with the theoretical ones as a function of 


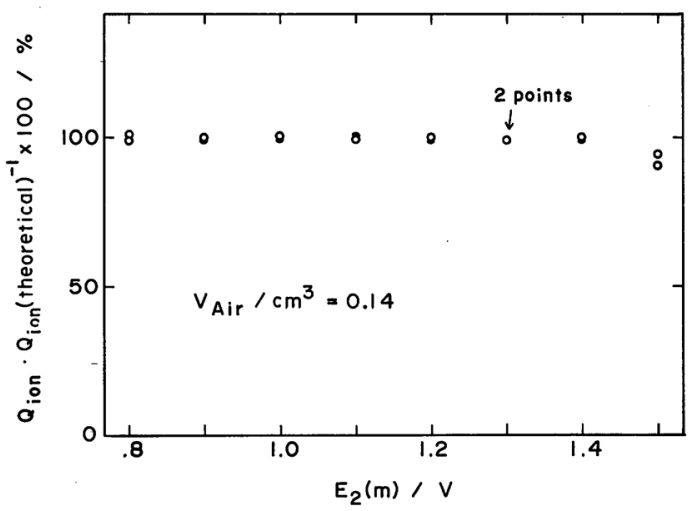

Fig. 7 Comparison of the measured $Q_{\text {ion }}$ values with the theoretical values as a function of $E_{2}(\mathrm{~m})$, under the conditions of $V_{\text {Air }} / \mathrm{cm}^{3}=0.14$ and a circulating Ar gas.

$E_{2}(\mathrm{~m})$ under the condition of $V_{\mathrm{Air}} / \mathrm{cm}^{3}=0.14$. When the $E_{2}(\mathrm{~m})$ value ranged from 0.8 to $1.4 \mathrm{~V}$, the agreement between these two values was excellent, (within 98.5\%). Even in the case of $E_{2}(\mathrm{~m}) / \mathrm{V}=1.5$, the difference was fairly small (less than $10 \%$ ). It was also found that $E_{2}(\mathrm{~m})$ had no effect on the time, $t^{\prime}$, for the current to return to the original value, $I_{\infty}$.

\section{(2) Oxygen analysis of lead}

The typical variation of the electrical current with time for two $\mathrm{Pb}$ (I) samples is presented in Fig. 8. A sample was dropped at the time in-

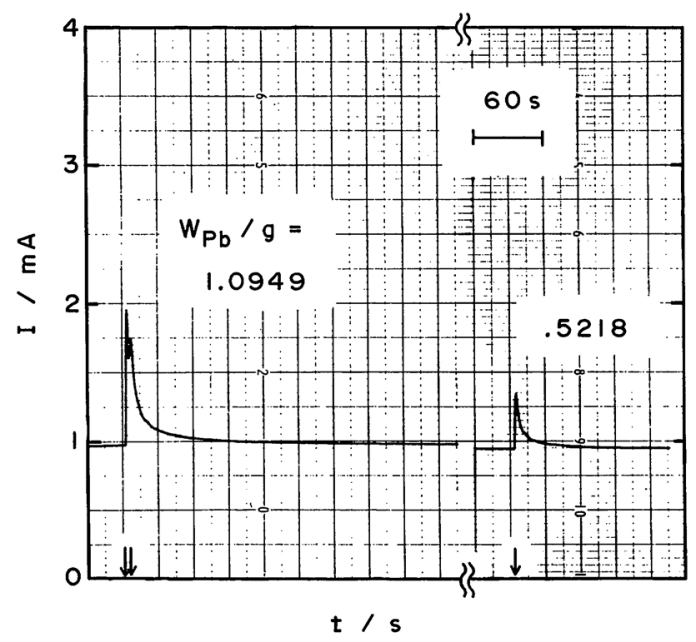

Fig. 8 Variation of the electrical current with time for lead (I) samples. The time at which the sample was dropped into the combustion chamber is indicated by $\downarrow$. dicated by $\downarrow$. As soon as the sample was dropped into the combustion chamber, the lead melted. Thus, the electrical current increased sharply at first and then returned to the original current, $I_{\infty}$, within $0.18 \mathrm{ks}$. Two peaks on the left-hand curve in Fig. 8 come from the fact that two pieces of the sample were dropped at slightly different times. The lead sample may decrease the temperature of the combustion chamber, $T_{2}$; however, in a preliminary experiment, it was observed that a change in $T_{2}$ had no effect on the background current, $I_{\infty}$. The $Q_{\text {ion }}$ values calculated from the measured $Q_{T}$ and $I_{\infty}$ values are shown in Fig. 9 as a function of the mass of the sample, $W_{\mathrm{Pb}}$. The uncertainty indicated by $I$ in this figure comes from the fact that the main contribution to the $Q_{T}$ values was from $I_{\infty}$. If the electrical current $I$ was stored in a computor and analyzed to determine the $Q_{\text {ion }}$ value, this uncertainty would disappear. As seen from Fig. 9, the measured $Q_{\text {ion }}$ values increased approximately linearly with the increase in $W_{\mathrm{Pb}}$. Using least squares, (under the constraint of $Q_{\text {ion }}=0$, for $W_{\mathrm{Pb}}=0$ ), a linear relationship was obtained, as indicated by the dashed line. From the slopes of these lines, the oxygen concentrations of $\mathrm{Pb}$ (I) and $\mathrm{Pb}$ (II) were determined; the results are $1.61 \times 10^{-4}$

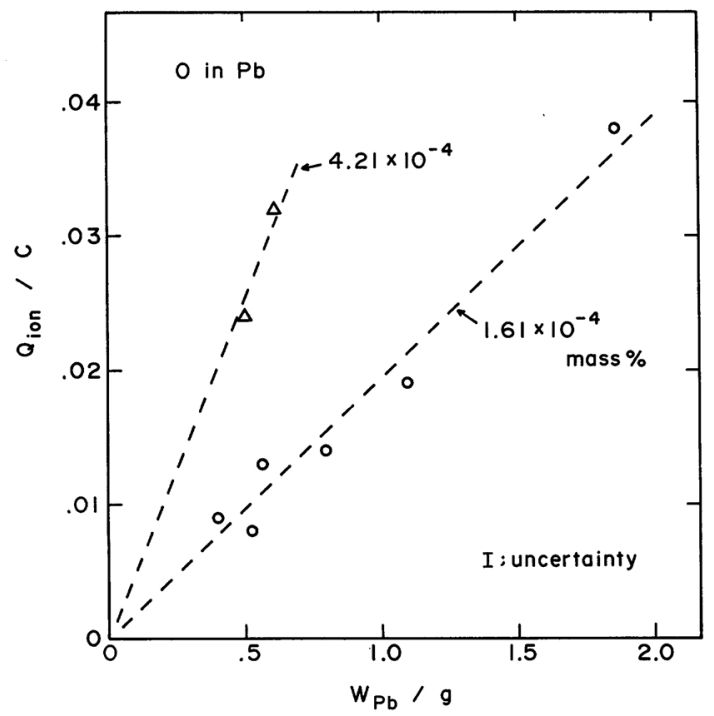

Fig. $9 Q_{\text {ion }}$ values measured for lead (I) and (II) as a function of $W_{\mathbf{P b}} \cdot--\mathrm{O}-\mathrm{:}$ lead (I), - $-\triangle--$ : lead (II). 
mass $\%\left(1.61\right.$ mass ppm) and $4.21 \times 10^{-4}$ mass $\%$ (4.21 mass ppm), respectively.

\section{Discussion}

An important feature appears in Fig. 4. The shape of the $I$ versus $t$ curve was weakly dependent of the amount of air. With the decrease in $V_{\mathrm{Air}}$, the electrical current returned to the original value, $I_{\infty}$, at a shorter time, $t^{\prime}$. Accordingly, one may speculate that a sharp peak on the curve, as indicated by $A$, is observed even for $V_{\text {Air }}$ much smaller than that adopted in the present investigation. As shown in Figs. 5 and 6 , the addition of hydrogen to the circulating Ar gas had no effect on the measured $Q_{\text {ion }}$ values. It should be emphasized that the buffer capacity of the circulating gas is increased several orders by the addition of hydrogen. These results imply that the oxygen analyzer of the present study can be applied to the analysis of inert gases containing a very low oxygen content. In conformity with this consideration, even when the oxygen concentration of lead samples were as low as 1 mass ppm, a steep peak was observed, as shown in Fig. 8. As far as this steep peak is observed, the oxygen content much less than 1 mass ppm can be determined using this oxygen analyzer.

When the oxygen concentration of metal samples is very low, the residual oxygen content in the sample becomes important. As previously stated, the analysis of lead (I) and (II) was performed under the conditions of $T_{1}=T_{2}=1073 \mathrm{~K}$ and $E_{2}(\mathrm{~m})=1.3 \mathrm{~V}$. According to thermodynamic calculations ${ }^{(5)(16)}$, the residual oxygen content in the molten lead after the analysis is negligibly small under these conditions, i.e., $2.3 \times 10^{-9}$ mass $\%\left(2.3 \times 10^{-5}\right.$ mass $\mathrm{ppm})$. Additionally, the residual oxygen content can be much reduced, when the temperature of the combustion chamber, $T_{2}$, is set to a lower value. For instance, it is $8.2 \times 10^{-11}$ mass $\%$ under the conditions of $T_{1} / \mathrm{K}=1073, T_{2} / \mathrm{K}=$ 873 and $E_{2}(\mathrm{~m}) / \mathrm{V}=1.3 . E_{2}(\mathrm{~m})$ may be set to a larger value, along with the adoption of a lower temperature of the cell (I), $T_{1}$. Under the conditions of $T_{1} / \mathrm{K}=973, T_{2} / \mathrm{K}=873$ and $E_{2}(\mathrm{~m}) /$ $\mathrm{V}=1.4$, the residual oxygen concentration in the molten lead is $7.3 \times 10^{-12}$ mass $\%$.

\section{Further considerations}

In the present investigation, the pump-out operation was performed by applying a voltage across wires $1^{\prime}$ and $2^{\prime}$ so that the emf across wires 1 and 2 had a preselected constant value, $E_{2}(\mathrm{~m})$. This emf, $E_{2}(\mathrm{~m})$, is related by the following equation to the oxygen chemical potentials at $x=0$ and $L$ :

$$
E_{2}(\mathrm{~m})=-(F / 4)\left(\mu_{\mathrm{O}_{2}}^{L}-\mu_{\mathrm{O}_{2}}^{\circ}\right)+I_{\text {ion }} \Omega_{\text {ion }}+I_{\text {ion }}^{\prime} \Omega_{\text {ion }},
$$

where $\Omega_{\text {ion }}$ is the ionic resistance of the solid electrolyte.

In a truely potentiostatic experiment, $I \Omega$ drop corresponding to the sum of the second and third terms in eq. (13) should be zero or constant so that $\mu_{\mathrm{O}_{2}}^{L}$ is constant. In fact, the highly reproducible and consistent results on $\gamma_{0}$, obtained in the previous modified coulometric titration experiments ${ }^{(4)-(8)(14)}$ have revealed that the $I \Omega$ drop is negligible because of the large ionic conductivity of the electrolyte used. Even if it was not so, the contribution by the term, $I_{\text {ion }}^{\prime} \Omega_{\text {ion }}$ can be neglected, because $I_{\text {ion }}^{\prime}$ is zero in the state $\mathrm{C}$ or virtually constant in the state B. When $I_{\text {ion }}$ changes with time from a highly large value to a small value, the contribution by $I_{\text {ion }} \Omega_{\text {ion }}$ may not be negligible. Because of a slight change in $\mu_{\mathrm{O}_{2}}^{L}, I_{\mathrm{e}}^{*}$ may be weakly dependent of time. However, in this case, the large value of $I_{\text {ion }}$ results in the large value of $Q_{\text {ion }}$. After all, the $Q_{\text {ion }}$ value evaluated using eq. (11) contains only a negligibly small error due to a drift in $I_{\mathrm{e}}^{*}$. When $I_{\text {ion }}$ is very small, the contribution by $I_{\text {ion }} \Omega_{\text {ion }}$ disappears. Accordingly, even a small value of $Q_{\text {ion }}$ does not contain any errors due to the terms, $\boldsymbol{I}_{\text {ion }} \boldsymbol{\Omega}_{\text {ion }}$ and $I_{\mathrm{ion}}^{\prime} \Omega_{\mathrm{ion}}$, in eq. (13). It should be mentioned that a conventional closed system may exhibit a very small leakage of oxygen from the atmosphere, which results in a slight increase in the background current, $I_{\infty}$. However, since this increase in $I_{\infty}$ appears to be constant, any error cannot be introduced by the slight leakage of oxygen.

As previously stated, the oxygen analyzer proposed in the present study has a strict theoretical basis. This technique could overcome the errors due to the release of oxygen from the 
electrolyte and electronic conduction in the electrolyte. Neither standard samples nor the establishment of blank conditions was necessary. The time spent for one run was as short as $0.18 \mathrm{ks}$. Electrical instruments are sensitive enough to detect a very small increment in electrical current very precisely. The current may be stored in a computor and analyzed to determine the $Q_{\text {ion }}$ value. In view of the case in which a $5 \mathrm{~g}$ sample produces an $I_{\text {ion }}$ of $10 \mu \mathrm{A}$ for $5 \mathrm{~s}$, the lower detection limit of this type of oxygen analyzer may be $10^{-3}$ mass ppm. Additionally, the solid electrolyte tube utilized in the cell (II) has been used for several months and may be used for more than a year, if the inside surface of the tube does not become contaminated. The oxygen analyzer developed in this study appears to be useful for inspection and quality control of materials produced on an industrial scale.

\section{Acknowledgements}

The authors wish to thank Mr. K. Hondoh for his assistance with the experiments. Nippon Kagaku Togyo Co. Ltd. and Seitetsu-Kagaku Co. Ltd. were kind enough to supply the stabilized zirconia electrolyte tubes and $\mathrm{Ar}+\mathrm{H}_{2}$ gas mixtures, respectively.

\section{REFERENCES}

(1) Y. Inoue: Collected Abstracts of the 1983 Fall Meeting of Japan Inst. Metals. p. 193.

(2) H. Kimura: Collected Abstracts of the 1983 Fall Meeting of Japan Inst. Metals, p. 195.

(3) S. Otsuka and Z. Kozuka: Abst. 8th ChubuKagaku-Kankeigakukyokai-Shiburengo, Fall Meeting, (Oct. 1977), p. 241, Nagoya, Japan.

(4) S. Otsuka and Z. Kozuka: Met. Trans. B, 10B (1979), 565.

(5) S. Otsuka: Molten Salts, 26 (1983), 129.

(6) S. Otsuka and Z. Kozuka: Met. Trans. B, 12B (1981), 501.

(7) S. Otsuka, H. Hanaoka and Z. Kozuka: Trans. JIM, 23 (1982), 563.

(8) S. Otsuka, Y. Kurose and Z. Kozuka: Trans. JIM, 24 (1983), 817.

(9) C. Wagner: Proc. Int. Comm. Electrochem. Thermo. and Kinetics (CITCE), 7th meeting, London, Butterworth Scientific Publication, London, (1957), p. 1955.

(10) C. Wagner: Z. Electrochem., 60 (1956), 4.

(11) C. Wagner: Z. Electrochem., 63 (1959), 1027.

(12) J. W. Patterson, E. C. Bogren and R. A. Rapp: J. Electrochem. Soc., 114 (1967), 752.

(13) S. Otsuka: Master Thesis, Faculty of Engineering, Osaka University, (1972).

(14) S. Otsuka, T. Sano and Z. Kozuka: Met. Trans. B, 11 B (1980), 313.

(15) S. Otsuka, Y. Matsumura and Z. Kozuka: Solid State Ionics, 3/4 (1981), 495.

(16) O. Kubaschewski and C. B. Alcock: Metallurgical Thermochemistry, 5th ed., Pergamon Press, Oxford, (1979), p. 380. 